\title{
Vascular trauma and lactate clearance as predictor determining mortality. Case report.
}

Authors: $\quad$ Carlos Andres C. Cortes Samaca, Hector Julio Melendez Florez, sonia margarita vivas garcia, Andrea Juliana Castillo Niño

Submitted: 10. December 2018

Published:

Volume:

Issue:

Affiliation:

Languages:

Keywords:

Categories:

DOI:

17. December 2018

5

10

Escuela de Medicina, Universidad Industrial de Santander, Bucaramanga, Colombia

Spanish, Castilian lactate, trauma, clearance, mortality

Medicine

10.17160/josha.5.10.512

\section{Abstract:}

Introduction: The serial measurement of serum lactate is essential for monitoring the therapeutic response of initial resuscitation and damage control in patients with vascular trauma, as well as a predictor of morbidity and mortality. Case report: We present a 25-year-old male patient who suffers trauma due to knife wound in the thorax with vascular injury, in whom a serial measurement of serum lactate, damage control and resuscitation was performed; subsequently, a vascular lesion was diagnosed, and vascular exploration of the subclavian vessels + arteriotomy was carried out due to the finding of vasospasm in the axillary artery with in situ thrombus and subclavian artery raffia. Conclusions: The serial measurement of serum lactate and its rapid clearance in the first hours of the event successfully predicts a low probability of morbidity and mortality despite the characteristics and severity of the trauma. (2015 The Authors. Published by the International Academy of Sciences, Humanities, and Arts under the terms of the Creative Commons Attribution License http://creativecommons.ora/licenses/bv/4.0/. which permits unrestricted use.

\section{JOSHA \\ Journal of Science, Humanities and Arts}




\title{
Reporte de caso
}

\section{Trauma vascular y depuración de lactado como predictor determinante de mortalidad. Reporte de caso.}

\section{Vascular trauma and lactate clearance as predictor determining mortality. Case report.}

\author{
Carlos Andrés Cortés Samacá (1), Hector Julio Melendez Florez (2). Sonia Margarita \\ Vivas Garcia (3), Andrea Juliana Castillo Niño
}

1 Medico y Cirujano, Especialista en Anestesiología y Reanimación, Departamento de Cirugía, Escuela de Medicina, Universidad Industrial de Santander, Bucaramanga, Colombia. Teléfono: 6651186. Fax: 6343125. Correo electrónico: andres1236@hotmail.com. Dirección de Correspondencia: Carrera 32 \# 29-31, Barrio Aurora.

2 Medico y Cirujano, Especialista en Anestesiología y Reanimación, Maestria en epidemiologia, Especialista en Medicina intensiva y cuidado crítico. Docente posgrado Anestesiologia UIS. Facultad de Salud. Escuela de Medicina, Universidad Industrial de Santander, Bucaramanga, Colombia. Fax: 6343 125. Dirección de Correspondencia: Carrera 32 \# 29-31, Barrio Aurora.

3 Medico General, Unidad de Cuidados Postanestesicos, Hospital Universitario de Santander, Bucaramanga, Colombia. Fax: 6350116. Correo electrónico: soniamargaritavivasgarcia@gmail.com. Dirección de Correspondencia: Carrera 30 \# 34-54, Barrio Aurora.

4 Medica y Cirujana. Facultad de Salud. Escuela de Medicina, Universidad Industrial de Santander, Bucaramanga, Colombia. Fax: 6343 125. Correo electrónico: julianacn2793@gmail.com. Dirección de Correspondencia: Carrera 32 \# 29-31, Barrio Aurora.

\section{RESUMEN}

Introducción: la medición seriada del lactato sérico es fundamental para el seguimiento de la respuesta terapéutica de reanimación inicial y control de daños en pacientes con trauma vascular, así como predictor de morbimortalidad. Caso clínico: Presentamos un paciente masculino de 25 años, quien sufre trauma por herida con cuchillo en tórax con lesión vascular, en quien se realizó medición seriada de lactato sérico, control de daños y reanimación hídrica; posteriormente se diagnostica lesión vascular, fue llevado a exploración vascular de vasos subclavios + arteriotomia por hallazgo de vasoespasmo en arteria axilar con trombo in situ y rafia de arteria subclavia. Conclusiones: la medición seriada del lactato sérico y su rápida depuración en las primeras horas del evento predice exitosamente una baja probabilidad de morbimortalidad a pesar de las características y la gravedad del trauma.

PALABRAS CLAVE: Trauma, depuración de lactato, lactato, lesión vascular

\section{SUMMARY}

Introduction: The serial measurement of serum lactate is essential for monitoring the therapeutic response of initial resuscitation and damage control in patients with vascular trauma, as well as a predictor of morbidity and mortality. Case report: We present a 25-year-old male patient who suffers trauma due to knife wound in the thorax with vascular injury, in whom a serial measurement of serum lactate, damage control and resuscitation was performed; subsequently, a vascular lesion was diagnosed, and vascular exploration of the subclavian vessels + arteriotomy was carried out due to the finding of vasospasm in the axillary artery with in situ thrombus and subclavian artery raffia. Conclusions: The serial measurement of serum lactate and its rapid clearance in the first hours of the event successfully predicts a low probability of morbidity and mortality despite the characteristics and severity of the trauma.

KEY WORDS: Trauma, clearance lactate, lactate, vascular injury.

\section{INTRODUCCIÓN}

El lactato sérico, como medida de la perfusión tisular ${ }^{1}$, se ha convertido desde mediados de la década de los noventa en un parámetro fundamental para el seguimiento de la respuesta terapéutica a la reanimación inicial y control de daños, así como predictor de mortalidad ${ }^{2}$, pero a diferencia de los pacientes con sepsis en quienes está ampliamente demostrado que los niveles elevados de lactato sérico se relacionan directamente con la mortalidad y morbilidad, se acepta que una depuración mayor del $20 \%$ en 6 horas mejora el pronóstico en los pacientes con trauma a pesar de que no existan suficientes estudios que determinen la depuración de lactato sérico como un eje central en la reanimación de este tipo de pacientes con trauma en estado de choque de origen hipovolémico con hipoperfusión secundaria. 


\section{CASO CLINICO}

Con la autorización y consentimiento informado del paciente, presentamos un caso de un paciente masculino de 25 años de edad, sin antecedentes patológicos, ni quirúrgicos, que llega al Hospital Universitario de Santander, por herida por arma corto punzante en región infra clavicular derecha con línea media clavicular. Al examen físico de ingreso paciente en malas condiciones generales, con alteración de su estado de conciencia, con palidez generalizada, con presión arterial: $45 / 30 \mathrm{mmHg}$, frecuencia cardiaca de 140 latidos por minuto, frecuencia respiratoria de 26 respiraciones por minuto. A la auscultación torácica con ruidos cardiacos rítmicos, taquicardicos, sin soplos e hipoventilación de todo el campo pulmonar derecho, con evidencia de herida en región infra clavicular derecha con línea media clavicular. Abdomen sin alteraciones, extremidades eutróficas con pulsos conservados y disminución de llenado capilar. Al examen neurológico somnoliento, con escala de Glasgow 9/15. Es valorado por el servicio de cirugía general, consideran paciente con hemotórax masivo, pasan 2 litros de Lactato Ringer, sin mejora de cifras tensionales, por lo que es llevado inmediatamente a cirugía. Al ingreso a salas de cirugía, se realiza monitoreo invasivo, se toman gases arteriales de ingreso que documentan acidemia metabolica con hiperlactatemia severa (valor de referencia normal de lactato sérico en gases arteriales: $<2 \mathrm{mmol} / \mathrm{dL}$ ) (ver tabla 1 ), se realiza inicialmente control de daños y se traslada a Unidad de Cuidados Posanestesicos (UCPA). En el posoperatorio inmediato se observa palidez, frialdad y disminución de pulsos en miembro superior derecho, se considera paciente con signos de lesión vascular y se realiza Angiotac con evidencia de interrupción de flujo arterial a nivel axilar en su tercio proximal, sin fuga del medio de contraste. Es llevado a segundo tiempo quirúrgico, donde se realiza exploración vascular de vasos subclavios + arteriotomia por hallazgo de vasoespasmo en arteria axilar con trombo in situ y rafia de arteria subclavia + inserción de catéter venoso central. El paciente es trasladado a UCPA, donde se toman nuevamente gases arteriales y se evidencia persistencia de acidemia metabolica con leve elevación lactato (ver tabla 1), por lo que se hace reanimación hídrica y soporte vasopresor, terapias orientadas por metas (tensión arterial, gasto urinario, llenado capilar, y depuración de lactato). A las 6 horas, se documenta una depuración de lactato asociado a mejora en el estado acido base del paciente y destete completo del soporte vasopresor. Paciente con buena evolución clínica, en posoperatorio descrito, quien al día siguiente es trasladado a salas de hospitalización general, con suplencia de oxigeno por sistema de bajo flujo estable hemodinamicamente, sin soporte vasopresor, con buen gasto urinario, con toracostomia permeable con drenaje hemático escaso. Al dia siguiente en salas generales, se hace control con gases arteriales de control sin trastorno acido base, adecuados índices de oxigenación y lactato dentro de límites normales (ver tabla 1).

El paciente no requirió nuevas intervenciones quirúrgicas y fue dado de alta diez días después del trauma, sin secuelas. 
Tabla 1. Seguimiento de gases arteriales y lactate en el tiempo.

\begin{tabular}{|c|c|c|c|c|c|c|}
\hline \multicolumn{7}{|c|}{ GASES ARTERIALES } \\
\hline \multirow{2}{*}{$\begin{array}{l}\text { FECHA } \\
\text { HORA }\end{array}$} & \multicolumn{3}{|c|}{ 13/11/16 } & \multicolumn{2}{|c|}{ 14/11/16 } & \multirow{2}{*}{$\frac{16 / 11 / 16}{15: 40}$} \\
\hline & 12:27 & $15: 18$ & 18:17 & 10:02 & $16: 10$ & \\
\hline TEMPERATURA & $36^{\circ} \mathrm{C}$ & $36^{\circ} \mathrm{C}$ & $37^{\circ} \mathrm{C}$ & $36^{\circ} \mathrm{C}$ & $36^{\circ} \mathrm{C}$ & $37^{\circ} \mathrm{C}$ \\
\hline PH & 7.135 & 7.229 & 7.326 & 7.391 & 7.444 & 7.412 \\
\hline $\mathrm{PCO} 2$ & 46 & 35.1 & 42 & 41.9 & 40.3 & 46.9 \\
\hline PO2 & 218 & 167 & 149 & 94.7 & 89.7 & 46.5 \\
\hline SAT 02 & $99.30 \%$ & $99 \%$ & $98.90 \%$ & $94.70 \%$ & $97.40 \%$ & $81.90 \%$ \\
\hline FIO2 & 0.6 & 0.35 & 0.35 & 0.32 & 0.28 & 0.21 \\
\hline $\begin{array}{l}\text { BICARBONATO } \\
\text { (mMol/L) }\end{array}$ & 15.1 & 14.3 & 21.4 & 24.8 & 27 & 29.2 \\
\hline LACTATO & 11.2 & 8.3 & 3.2 & 2.7 & 1.3 & 1.1 \\
\hline
\end{tabular}

\section{Trauma}

El trauma ha sido identificado como la segunda causa de muerte en Colombia, posterior a enfermedades cardiovasculares, responsable de 15.747 muertes en el 2012, donde las lesiones por arma de fuego y las lesiones por armas corto punzantes encabezan el listado, causando 12.285 y 2.475 muertes, respectivamente ${ }^{3}$. Pero no solo es un problema a nivel de Colombia, la Organización Mundial de la Salud estimó que, en el año 2000, 5 millones de personas murieron en circunstancias violentas y la mayoría de estas muertes ocurrieron en países en vía de desarrollo ${ }^{4}$.

La causa de la muerte en trauma durante las primeras horas es generalmente de origen hemorrágico ${ }^{1,2}$, superado sólo por Trauma Cráneo Encefálico ${ }^{5-6}$, sin embargo el choque hipovolémico tiene la ventaja que responde a la terapéutica que se instaura en la mayoría de las instituciones de salud que se adhieren a las recomendaciones de reanimación inicial con cristaloides y hemoderivados, para mantener una presión arterial sistólica normal, en el marco de la estrategia Advanced Trauma Life Support (ATLS) ${ }^{5,7}$, disminuyendo entre un $30 \%$ a $40 \%$ la morbimortalidad ${ }^{6}$.

\section{Evaluacion clinica vs. Medicion de lactato en sangre}

La dificultad que se ha encontrado en la evaluación y el seguimiento a las intervenciones terapéuticas en la reanimación inicial con los signos vitales como se recomienda en $\mathrm{ATLS}^{7}$, es que se ha demostrado que tanto la presión arterial como la frecuencia cardiaca pueden llegar a ser imprecisas y tener dificultades técnicas, además debido a los mecanismos neurohumorales como respuesta a la hemorragia pueden estar dentro de parámetros normales incluso en estados avanzados de depleción de volumen intravascular, hipoperfusión e hipoxia ${ }^{7-8}$. Es por esto que ha surgido la necesidad de 
estandarizar pruebas más confiables para evidenciar de forma dinámica el estado de perfusión del paciente ${ }^{8}$.

Conociendo la fisiopatología del choque hemorrágico, se sabe que una caída del volumen intravascular desencadena una respuesta sistémica predecible, mediada por cambios vasculares locales y modificaciones en el sistema neuroendocrino por estimulación

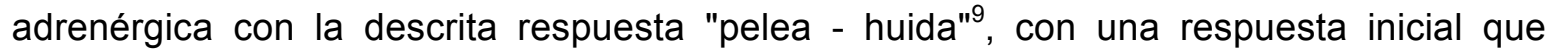
genera vasoconstricción en órganos con aceptable tolerancia a la isquemia (musculo, grasa, piel) para preservar el flujo sanguíneo en órganos vitales, principalmente corazón y cerebro.

\section{Fisiopatologia}

Si estos mecanismos anteriormente descritos son insuficientes para garantizar la homeostasis del paciente, por persistencia de la noxa causal o por reanimación insuficiente, el paciente experimenta cambios en su medio interno, instaurándose la denominada "Triada de la muerte", que consiste en acidosis, coagulopatía e hipoperfusión ${ }^{10}$. La hipoperfusión, con la reducción secundaria del oxígeno a nivel celular, lleva a un metabolismo anaeróbico a la célula, lo que hace que se acumule piruvato ante la falta de eficiencia de la enzima piruvato deshidrogenasa para convertirlo en acetil-CoA. El exceso de piruvato se convierte en lactato por reacción a través de la enzima lactato deshidrogenasa ${ }^{13}$. El lactato, con un $\mathrm{pK}_{\mathrm{a}}$ de 3.8 aproximadamente, se libera de los tejidos acompañados por un protón, y a un $\mathrm{pH}$ por encima de 6 , el ácido láctico se disocia completamente entonces la producción de lactato excesiva puede dar lugar a acidosis láctica con todas las complicaciones que conocemos, efecto inotrópico negativo, vasoplejía, alteración de la disociación de la hemoglobina, reducción de la efectividad de los fármacos vasopresores, disminución de la perfusión esplácnica y alteración de la tasa de filtración glomerular, entre otros ${ }^{10}$.

De esta manera, empezaron a surgir una gran cantidad de estudios desde la década de los noventa, donde se demostró que no solo el nivel elevado de lactato sérico al ingreso, sino su aclaramiento en las primeras 24 horas $^{1,2}$, están relacionados directamente con la morbimortalidad de los pacientes traumatizados.

\section{DISCUSIÓN}

Se presenta el caso de un paciente adulto joven, sin comorbilidades, quien ingresa al Hospital Universitario de Santander, con un estado de choque hipovolémico en el contexto de trauma por herida corto punzante en tórax con hipoperfusión, en quien se realiza el abordaje de manejo inicial de urgencias recomendado por el ATLS, y posterior manejo de control de daños, reanimación dirigida por metas y posterior estabilización, en segundo tiempo quirúrgico reparación anatómica de daños. En este paciente se realizó un seguimiento del lactato sérico reportado en gases arteriales. En el primer reporte, realizado al ingreso, se encontró hiperlactatemia (Lactato 11,2 $\mathrm{mMol} / \mathrm{L}$ ), lo que de forma 
aislada y según los estudios publicados en sepsis severa extrapolados a trauma, se relaciona con el desarrollo de falla orgánica múltiple y una mortalidad tan alta como $89 \%$, teniendo en cuenta que la determinación de lactato sérico es útil como marcador del metabolismo aerobio secundario a hipoperfusión tisular ${ }^{11}$. En trauma se ha encontrado que aunque el monitoreo de lactato al ingreso es bastante útil para predecir desenlaces, la tendencia del monitoreo seriado es una valiosa aproximación de mortalidad hospitalaria ${ }^{8}$. Revisando la literatura publicada sobre el tema, vale la pena destacar la revisión sistemática de $\mathrm{Kruse}^{8}$, quien recopila los estudios realizados hasta el 2011, donde encuentra 8 estudios retrospectivos de moderada calidad, en los que se evidencia la utilidad de la medición y seguimiento de lactato sérico como predictor de mortalidad. También se destaca el estudio retrospectivo de Shapiro ${ }^{12}$, autoridad mundial en tema de perfusión y microcirculación, donde plantea el lactato inicial y la depuración de lactato sérico a las 6 horas, como un predictor independiente de mortalidad en pacientes con trauma. En un estudio de cohorte prospectivo observacional ${ }^{13}$, Riu considera que la depuración temprana de lactato, definido por el autor como el aclaramiento a las 2 y 4 horas, es una importante variable pronóstica independiente y este autor, al igual que otras publicaciones $^{14,15}$, plantean que deben realizarse más estudios, de carácter prospectivo, para que en un futuro se pueda contar con adecuada evidencia científica para incorporar en las guías de toma de decisiones para la reanimación guiada por metas, la depuración temprana de lactato en los pacientes de trauma.

En el caso analizado se evidenció una rápida depuración de lactato sérico (Figura $\mathrm{N}^{\circ} 1$ ). Se encontró que el paciente depuró un $25.8 \%$ del lactato en las primeras 3 horas y $71.4 \%$ a las 6 horas, lo que predijo exitosamente una baja probabilidad de mortalidad a pesar de las características y la gravedad del trauma.

Figura 1. Depuracion de lactato en el tiempo

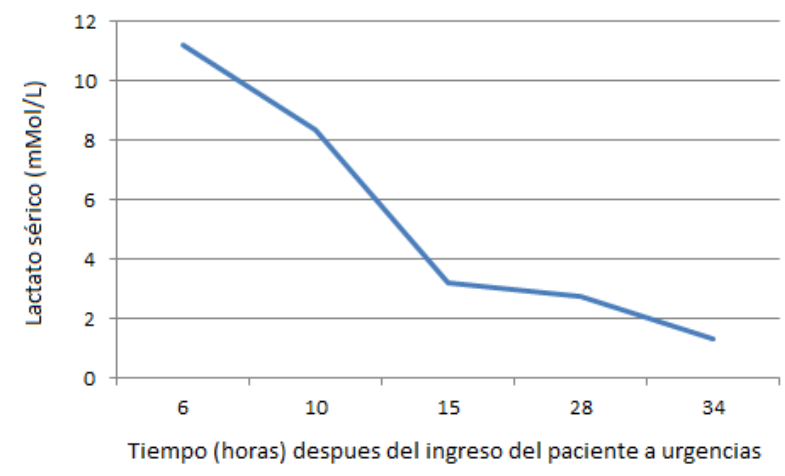

\section{CONCLUSION}

Si bien hay aceptación en los beneficios de la depuración temprana de los niveles de lactato sérico elevados y como variable pronostica independiente ${ }^{16-17}$, no hay disponibles suficientes estudios de alta calidad que determinen la depuración del lactato sérico como 
un eje central en la reanimación en trauma ${ }^{17}$, los distintos autores concluyen que se deben realizar estudios de carácter prospectivo, para determinar sí se puede extrapolar la experiencia que se tiene $\operatorname{sepsis}^{18}$ a los pacientes traumatizados, ya que podría usarse como un marcador pronostico sensible y de bajo costo en este tipo de pacientes. Actualmente se esta desarrollando un estudio de cohorte de carácter prospectivo en nuestra institución.

Financiación

Ninguna.

Conflicto de intereses

Ninguno.

\section{REFERENCIAS}

1. Baranov D, Neligan P. Trauma and Aggressive Homeostasis Management. Anesthesiology Clin [Internet]. (2007) mar; 25(1):49-63. Disponible en: www.anesthesiology.theclinics.com/article/S08898537(06)00093-9/pdf

2. Manikis P1, Jankowski S, Zhang H, Kahn RJ, Vincent JL. Correlation of Serial Blood Lactate Levels to Organ Failure and Mortality After Trauma. Am J Emerg Med [Internet]. (1995) nov; 13(6):619-22. Disponible en: www.ajemjournal.com/article/0735-6757(95)90043-8/pdf.

3. Instituto Nacional de Salud [Internet]. Colombia: INS; c 2014, Boletín No. 4 [Acceso 01 de agosto de 2015]. Disponible en: http://www.ins.gov.co/lineas-de accion/ons/boletin4/interactivo/tasasM.html.

4. Krug E, Dahlberg L, Mercy J, Zwi A, Lozano R. World health report on violence and health. Geneva: World Health Organization. 2002 disponible en http://www.who.int/violence_injury_prevention/violence/world_report/en/summary_en.pdf?ua=1

5. Dutton RP, Mackenzie CF, Scalea TM. Hypotensive Resuscitation during Active Hemorrhage: Impact on In-Hospital Mortality. J Trauma [Internet].2002 jun ;52 (6):1141-1146. disponible en: https://em.osumc.edu/education/journalClub/hypotensiveresuscmortality.pdf

6. Kauvar DS, Lefering R, Wade CE. Impact of Hemorrhage on Trauma Outcome: An Overview of Epidemiology, Clinical Presentations, and Therapeutic Considerations. J Trauma [Internet]. 2006 jun ;60:S3-S11

7. American College Of Surgeons [Internet]. Chicago: ACS; Capítulo 1: Evaluación y Manejo Inicial. Manual del Estudiante: Curso en Advanced Trauma Life Support@. Ed 9². c 2012. P. 2-22. Disponible en: https://es.scribd.com/doc/270103683/ATLS-9-Edicion-Espanol

8. Kruse $\mathrm{O}^{1}$, Grunnet $\mathrm{N}$, Barfod $\mathrm{C}$. Blood lactate as a predictor for in-hospital mortality in patients admitted acutely to hospital: a systematic review. Scand J Trauma Resusc Emerg Med. 2011 Dec 28;19:74 disponible en: https://sjtrem.biomedcentral.com/articles/10.1186/1757-7241-19-74

9. Dutton RP. Current Concepts in Hemorrhagic Shock. Anesthesiology Clin [internet] . 2007 Mar;25(1):23-34.disponible en: www.anesthesiology.theclinics.com/article/S0889-8537(06)00098$8 /$ pdf.

10. Régnier MA, Raux M, Le Manach $Y$ et al. Prognostic significance of blood lactate and lactate clearance in trauma patients. ANESTHESIOLOGY [Internet]. 2012 dec; 117:1276-88 .disponible en: http://anesthesiology. pubs.asahq.org/article. aspx?articleid $=1934409$

11. Jasso-Contreras $\mathrm{G}$ et al. Lactato sérico como predictor de mortalidad. Rev Med Inst Mex Seguro Soc. 2015;53(3):316-21. Disponible en: http://www.medigraphic.com/pdfs/imss/im-2015/im153m.pdf

12. Odom SR, Howell MD, Silva GS et al. Lactate clearance as a predictor of mortality in trauma patients. J Trauma Acute Care Surg [Internet]. 2014 Mar; 76(3):902.disponible en: 
http://journals.Iww.com/jtrauma/Citation/2014/07000/Lactate_clearance_as_a_predictor_of_mortality. 33.aspx

13. Schnüriger $B$, Talving $P$, Inaba $K$ et al. Biochemical Profile and Outcomes in Trauma Patients Subjected to Open Cardiopulmonary Resuscitation: A Prospective Observational Pilot Study. World J Surg [Internet].(2012) aug; 36:1772-1778 Disponible en: http://link.springer.com/article/10.1007\%2Fs00268-012-1583-3

14. Laverde CE, Correa AF, Joya AY. Lactato y déficit de bases en trauma: Valor Pronóstico. Rev Colomb Anestesiol 2014 mar;42:60-4 disponible en: http://www.revcolanest.com.co/es/lactato-deficitbases-trauma-valor/articulo/S0120334713000750/

15. Gunnerson K, Saul M, He S, et al. Lactate versus non-lactate metabolic acidosis: a retrospective outcome evaluation of critically ill patients. Crit Care v.10(1); 2006. Disponible en: https://www.ncbi.nlm.nih.gov/pmc/articles/PMC1550830/

16. Okello $M$, Makobore $P$, Wangoda $R$ et al. Serum lactate as a predictor of early outcomes among trauma patients in Uganda. Int J Emerg Med [Internet]. (2014) , 7:20. Disponible en: www.ncbi.nlm.nih.gov/pmc/articles/PMC4105872/

17. Husain FA, Martin MJ, Mullenix PS et al. Serum lactate and base deficit as predictors of mortality and morbidity. Am J Surg [Internet]. (2003) may;185:485-91. Disponible en: www.americanjournalofsurgery.com/article/S0002-9610(03)00044-8/pdf.

18. Dellinger RP, Levy MM, Rhodes A et al. Surviving Sepsis Campaign: International Guidelines for Management of Severe Sepsis and Septic Shock, 2012. Intensive Care Med [Internet]. (2013) feb;39(2):165-228.Disponible en: link.springer.com/article/10.1007/s00134-012-2769-8. 